\title{
EMISSÕES EM MOTOCICLOS: PRIMEIRO ENSAIO DE PROFICIÊNCIA
}

\author{
Paulo R. M. Silva ${ }^{1}$, Valnei S. Cunha ${ }^{1}$, Werickson F. C. Rocha ${ }^{1}$, Gabriel F. \\ Sarmanho ${ }^{1}$, Joyce C. Andrade ${ }^{2}$ and Marcello Depieri ${ }^{3}$ \\ ${ }^{1}$ Inmetro - Divisão de Metrologia Química \\ ${ }^{2}$ Inmetro -Divisão de Comparações Interlaboratoriais e Ensaios de Proficiência \\ ${ }^{3}$ Magneti Marelli Automotive Systems - Powertrain \\ E-mails: \\ prsilva@inmetro.gov.br, \\ vscunha@inmetro.gov.br, \\ wfrocha@inmetro.gov.br, \\ gfsarmanho@inmetro.gov.br, \\ icandradre@inmetro.gov.br, marcello.depieri@magnetimarelli.com
}

\section{RESUMO}

Um Ensaio de Proficiência (EP) é uma ferramenta de qualidade poderosa na comparação de resultados analíticos obtidos em condições similares. O principal objetivo do EP é o de avaliar a competência técnica dos laboratórios participantes. Dando continuidade a parceria AEA-Inmetro, pela primeira vez um teste de proficiência foi conduzido para análise de emissões em motociclos. Sete laboratórios participaram deste EP.

Os parâmetros analisados foram os seguintes: $\mathrm{CO}, \mathrm{CO}_{2}, \mathrm{NOx}$, THC, aldeídos totais (opcional) e autonomia. Os testes foram realizados conforme a diretiva 97/24/CE, NBR 12026 para determinação de aldeídos e NBR 7024 para determinação da autonomia. Dois motociclos foram utilizados neste EP: Um em ciclo urbano movido a etanol hidratado de referência e outro em ciclo urbano e extraurbano movido a gasolina A22\%.

Para verificar se havia alguma diferença estatística na distribuição da população, as médias dos resultados de cada laboratório foram comparadas usando o teste de Kruskal-Wallis. Os Outliers foram avaliados com a utilização de box-plot. Para este primeiro EP em motociclos, o objetivo principal foi o de comparar os resultados obtidos de cada laboratório e não o de avaliar o desempenho dos laboratórios participantes deste primeiro ensaio de proficiência.

Para ambos os motociclos avaliados, observaram-se diferenças significativas nas distribuições das populações para todos os parâmetros analisados, sendo que para - motociclo movido à gasolina, foi observado um outlier para o parâmetro "autonomia". Para a moto movida a etanol foram observados outliers para os parâmetros $\mathrm{CO}_{2}$ e autonomia.

\section{INTRODUÇÃO}

O problema da poluição do ar constitui um sério problema à saúde humana, diminuindo a sua qualidade de vida. Veículos automotivos são um fonte potencial deste tipo de poluição, visto que a frota brasileira de carros dobrou na última década, 
enquanto que a frota de motos quadriplicou [1]. Faz-se então necessário que os níveis de emissão de motos sejam avaliados [2], como já o são para automóveis [3].

A análise dos poluentes por um laboratório é um dos itens mais delicados de um ensaio de emissão e uma das formas de garantir que os resultados analíticos obtidos são aceitáveis é participação em ensaios de proficiência (EP). Ele propicia subsídios aos laboratórios na identificação e solução de problemas analíticos, contribuindo para a harmonização de metodologias e resultados obtidos.

Um ensaio de proficiência tem por objetivo comparar os resultados de diferentes laboratórios obtidos em condições similares e também o de fazer uma avaliação contínua do desempenho dos diferentes laboratórios participantes [4,5]. Este processo é bem estabelecido para as análises de emissão em carros, já que há cinco rodadas finalizadas desde 2004 [6-8], mas é a primeira vez que tal ensaio é conduzido para motociclos. De posse do relatório final, cada participante poderá rever os processos de análises, assim como implementar as melhorias necessárias.

Neste trabalho apresentamos os resultados obtidos para este primeiro ensaio de proficiência realizado em motociclos. Neste teste foram determinados os seguintes parâmetros: $\mathrm{CO}, \mathrm{CO}_{2}, \mathrm{NO}_{x}, \mathrm{THC}$, aldeídos totais e autonomia. Para observação da distribuição das populações, as médias dos resultados obtidos por cada laboratório foram comparadas pelo teste de Kruskal-Wallis. Para a visualização das distribuições dos resultados e detecção de outliers, o box-plot foi utilizado.

\section{METODOLOGIA}

A metodologia do Ensaio de Proficiência foi estabelecida em protocolo [9] onde as características dos motociclos de teste, a logística de transporte, as datas de análise e a definição dos parâmetros determinados foram definidas em acordo com todos os participantes do processo. Para este primeiro EP, dois motociclos diferentes foram utilizados, a saber: motociclo de 149 cilindradas movido a etanol (EHR) em ciclo urbano e outro de 250 cilindradas movido a gasolina A22\% em ciclos urbano e extraurbano limitados aos $120 \mathrm{~km} / \mathrm{h}$.

Sete laboratórios participaram deste primeiro EP de emissões em motociclos; Cada participante recebeu um código para assegurar a confidencialidade do processo. Os parâmetros analisados foram monóxido de carbono (CO), dióxido de carbono $\left(\mathrm{CO}_{2}\right)$, óxidos de nitrogênio $\left(\mathrm{NO}_{\mathrm{x}}\right)$, hidrocarbonetos totais $(\mathrm{THC})$, autonomia urbana e aldeídos totais. Para este último parâmetro, a participação foi opcional e os laboratórios participantes receberam uma codificação diferente.

Os laboratórios realizaram os testes de acordo com a diretiva 97/24/CE, a norma ABNT NBR 12026 para determinação de aldeídos e a norma ABNT NBR 7024 para determinação de autonomia. Os resultados obtidos expressam a quantidade de poluentes emitida para um veículo leve do ciclo Otto $\left(\mathrm{CO}, \mathrm{CO}_{2}, \mathrm{NO}_{\mathrm{x}}, \mathrm{THC}\right.$ e aldeídos) expressos em $\mathrm{g} / \mathrm{km}$ e autonomia urbana $\mathrm{em} \mathrm{km} / \mathrm{L}$.

A comparação das médias dos participantes foi efetuada utilizando o teste de Kruskal-Wallis para cada um dos parâmetros analisados. O teste é baseado nas seguintes hipóteses: $\mathrm{H}_{0}$ - não há diferença nas distribuições das populações; $\mathrm{H}_{\mathrm{a}}-$ Há diferença na distribuição. A equação 1 mostra a estatística da equação. 


$$
H=\frac{12}{N(N+1)}\left(\frac{R_{1}^{2}}{n_{1}}+\frac{R_{2}^{2}}{n_{2}}+\ldots+\frac{R_{k}^{2}}{n_{k}}\right)-3(N+1)
$$

Onde $\mathrm{n}_{\mathrm{i}}$ é número de repetições de cada laboratório, $\mathrm{N}$ é o número total de laboratórios, $\mathrm{R}_{\mathrm{i}}$ é a soma dos postos para cada laboratório e $\mathrm{H}$, a estatística de Kruskal-Wallis.

Se algum parâmetro mostrou diferença significativa na distribuição, foram identificados quais pares são diferentes estatisticamente, conforme mostrado na equação 2.

$$
\left|\overline{R_{j}}-\overline{R_{j}}\right| \leq Z_{\alpha / k(k-1)} \sqrt{\frac{N(N+1)}{12}\left(\frac{1}{n_{j}}+\frac{1}{n_{i}}\right)}
$$

Onde $\mathrm{N}$ é igual ao número total de resultados obtidos, $\mathrm{n}$ é o número de replicatas de cada laboratório, k é o número de laboratórios participantes do EP, $\alpha$ é o nível de confiança (com $95 \%$ de confiança).

O Box-plot (gráfico de caixa) [10,11] é um gráfico utilizado para visualização da distribuição de um conjunto de dados. É formado por cinco medidas: $1^{\circ}$ quartil (Q1), $3^{\circ}$ quartil (Q3), mediana (Q2), valor mínimo (LI) e valor máximo (LS). Além disso, o box-plot pode ser empregado para avaliação de outliers. Dessa forma, os valores mínimo e máximo são calculados de acordo com as equações 3 e 4.

$$
\begin{aligned}
& \mathrm{LI}=\mathrm{Q} 1-1,5^{\star}(\mathrm{Q} 3-\mathrm{Q} 1) \\
& \mathrm{LS}=\mathrm{Q} 3+1,5^{\star}(\mathrm{Q} 3-\mathrm{Q} 1)
\end{aligned}
$$

Se o valor obtido > LS ou o valor obtido < LI, o valor obtido é considerado um outlier;

Se $\mathrm{LI} \leq$ o valor obtido $\leq \mathrm{LS}$, o valor obtido não é considerado um outlier;

Onde, o valor obtido é o resultado de cada medição de cada parâmetro por participante.

\section{RESULTADOS}

\section{Motociclo movido a gasolina}

A figura 1(a-f) mostra a distribuição das médias dos resultados para cada parâmetro avaliado neste EP. As linhas sólidas correspondem às médias dos resultados e as pontilhadas, a mediana. Em todos os casos, para um nível de $95 \%$ de confiança, foram observadas diferenças significativas na distribuição das populações $\left(p_{\text {calculado }}<0,05\right)$. Para o parâmetro aldeído (figura 1-f), somente três laboratórios enviaram os resultados das análises. A avaliação da diferença entre os pares de média foi feita utilizando a equação 2 para identificar quais seriam as médias específicas dos participantes que diferem dos outros. Para a determinação de $\mathrm{CO}$, os pares 21-98 e 93-98 apresentaram diferenças significativas entre suas médias. Para $\mathrm{CO}_{2}$, o par 81-98 mostrou o mesmo comportamento, enquanto que para a determinação de THC o par 93-98 mostrou diferenças significativas entre as médias. Já para o parâmetro $\mathrm{NO}_{\mathrm{x}}$, ० par 21-93 mostrou diferenças estatísticas 
significativas. Para o parâmetro aldeídos totais, as médias dos laboratórios AT-70 e AT-96 são estatisticamente diferentes. Finalmente os outliers foram avaliados, conforme mostrado na figura 2. Com exceção do parâmetro autonomia, onde o laboratório 98 (figura 2-e) mostrou resultados acima do limite superior calculado, não foram observados outros outliers.
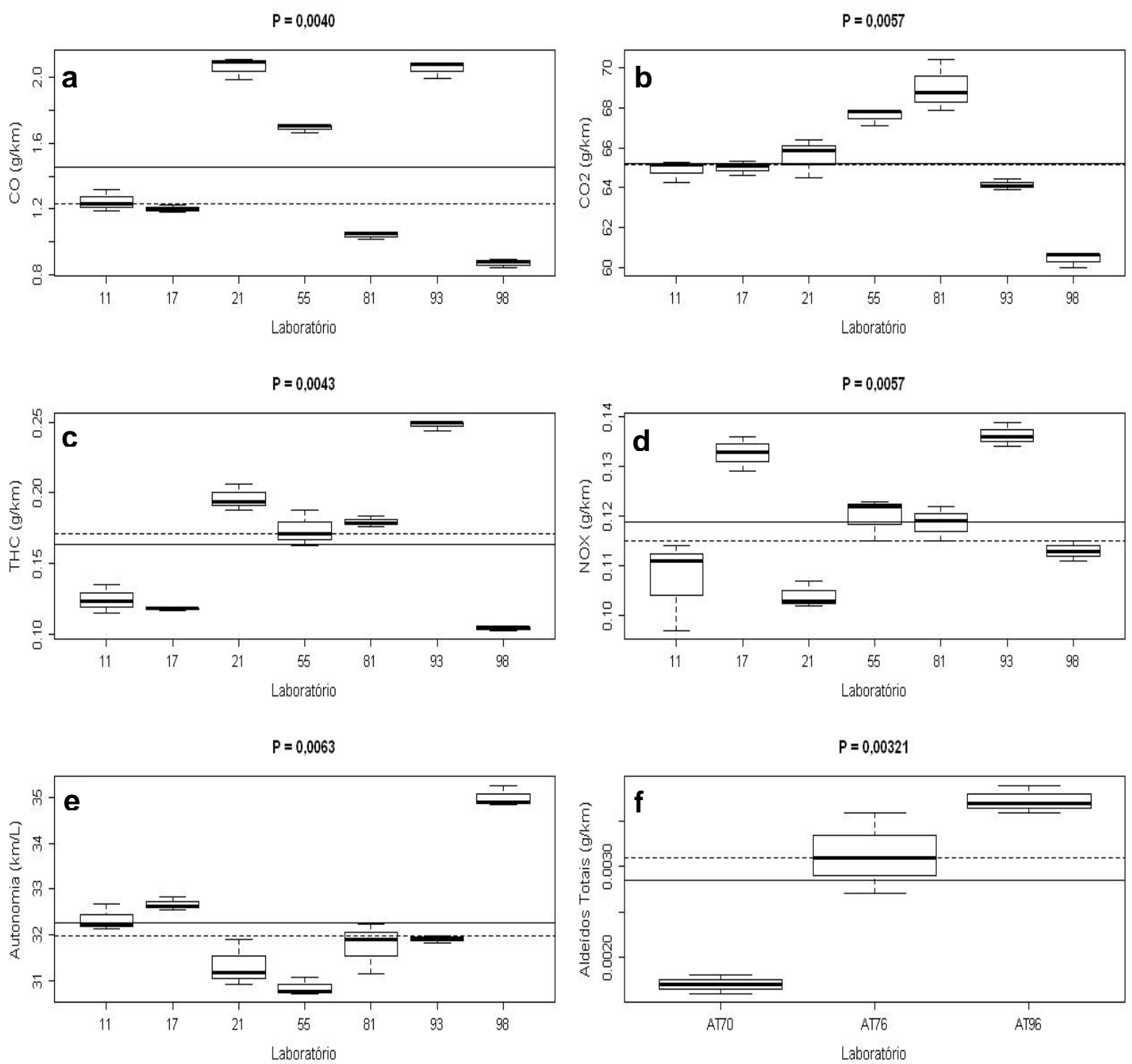

Figura 1. Distribuição dos resultados para o teste de proficiência de emissões em motociclo movido a gasolina. (a) $\mathrm{CO}$, (b) $\mathrm{CO}_{2}$, (c) $\mathrm{THC}$, (d) $\mathrm{NO}_{\mathrm{x}}$, (e) autonomia e (f) aldeídos totais 


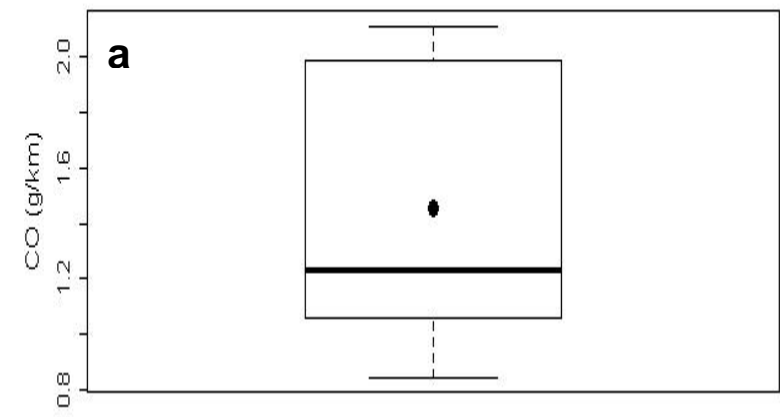

Laboratórios

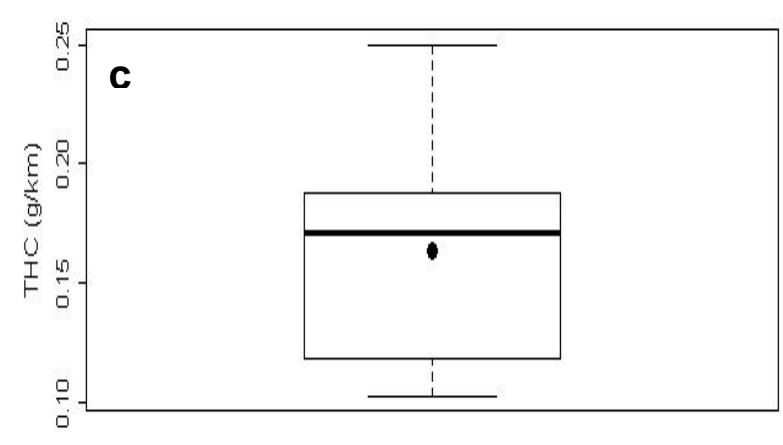

Laboratórios

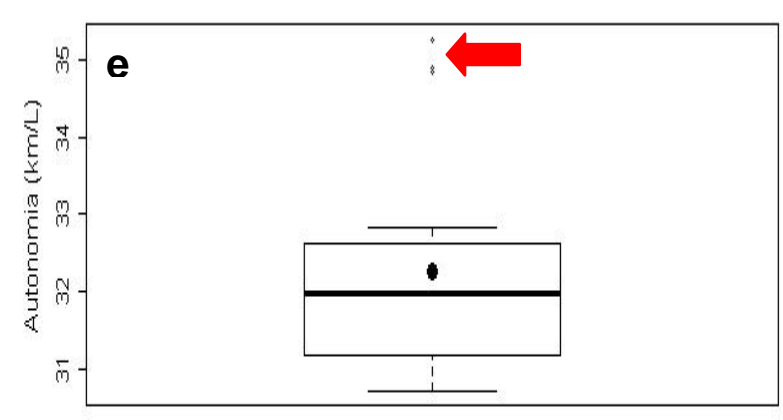

Laboratórios

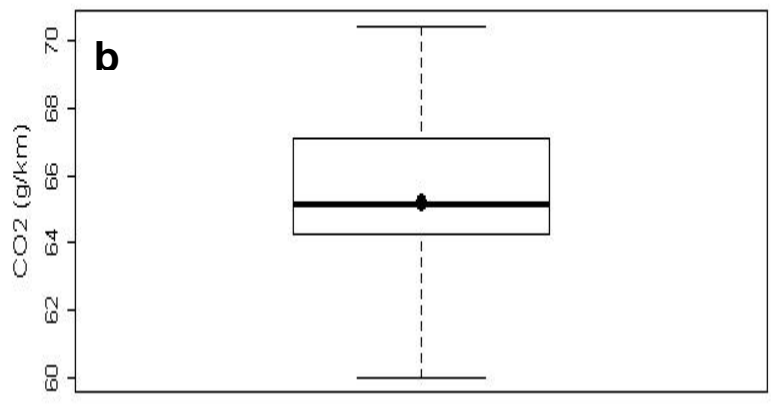

Laboratórios

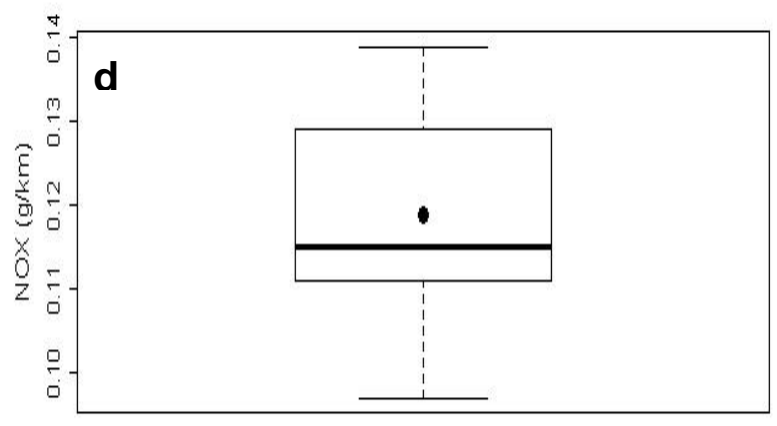

Laboratórios

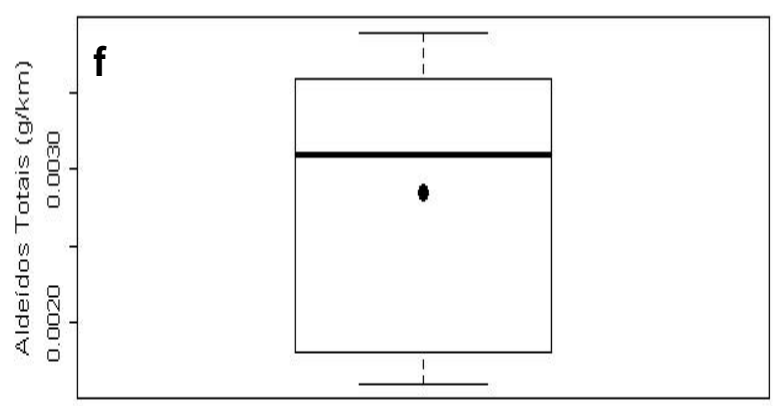

Laboratórios

Figura 2. Box-Plot para o teste de proficiência de emissões em motociclo movido a gasolina. (a) $\mathrm{CO}$, (b) $\mathrm{CO}_{2}$, (c) $\mathrm{THC}$, (d) $\mathrm{NO}_{x}$, (e) autonomia e (f) aldeídos totais.

\section{Motociclo movido a etanol}

Como mostrado na figura 3 (a-f) para o motociclo movido a etanol, foi observado uma diferença estatística significativa na distribuição da população. Para a determinação de $\mathrm{CO}$, os laboratórios 11-21 e 11-81 mostraram diferenças estatísticas significativas entre suas médias. Para $\mathrm{CO}_{2}$ o par 81-98 mostrou o 
mesmo comportamento, enquanto que para a determinação de THC o par 21-55 mostrou diferenças significativas entre suas médias, enquanto que para o parâmetro $\mathrm{NO}_{x}$, o par 17-81 as médias são estatisticamente diferentes. Para a análise de autonomia, dois pares apresentaram diferenças estatísticas, 11-81 e 81-93. Para aldeídos totais, as médias dos laboratórios AT-96 e AT-96 diferem estatisticamente.

Como mostrada na figura 4, o laboratório 81 apresentou outliers para $\mathrm{CO}_{2}$ (figura 4-b) e autonomia (4-e). Para o primeiro parâmetro, os valores obtidos estavam acima do limite superior, enquanto que para autonomia, os resultados apresentaram um comportamento oposto, abaixo do limite inferior.
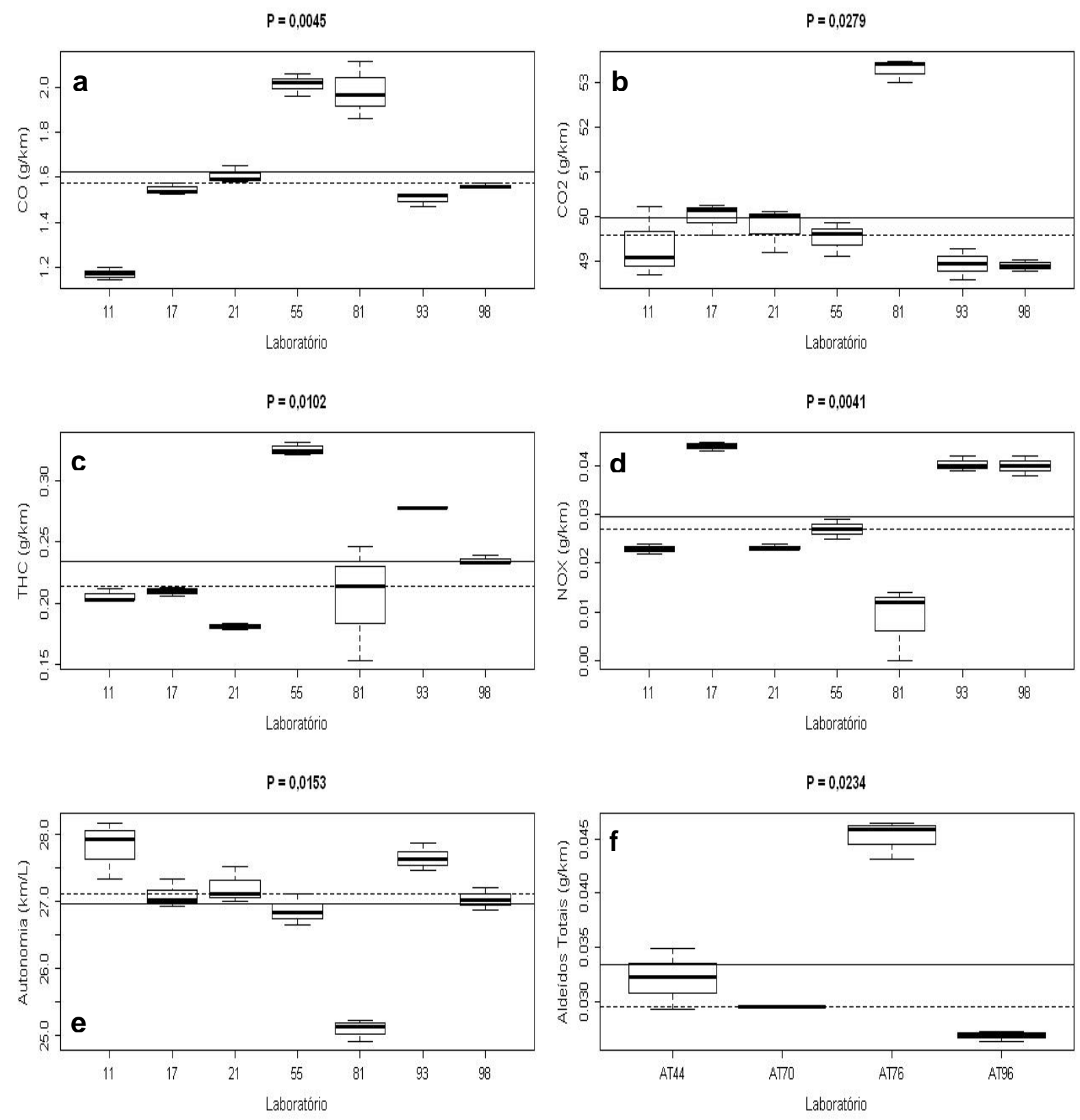

Figura 3. Distribuição dos resultados para o teste de proficiência de emissões em motociclo movido a etanol. (a) $\mathrm{CO}$, (b) $\mathrm{CO}_{2}$, (c) $\mathrm{THC}$, (d) $\mathrm{NO}_{\mathrm{x}}$, (e) autonomia e (f) aldeídos totais 


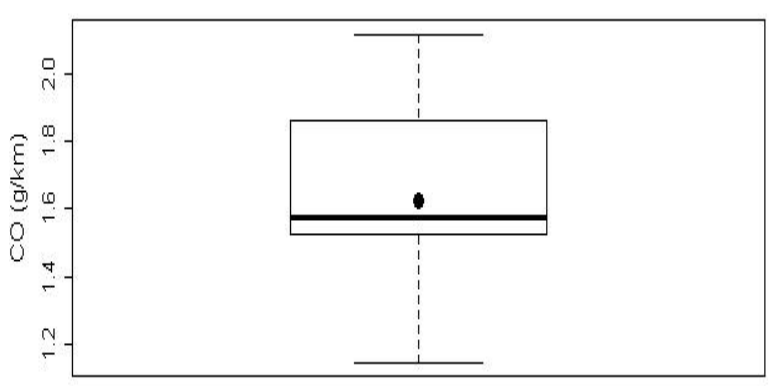

Laboratórios

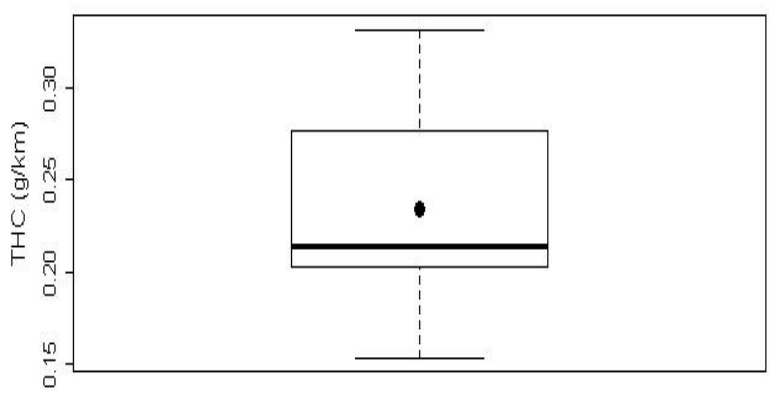

Laboratórios

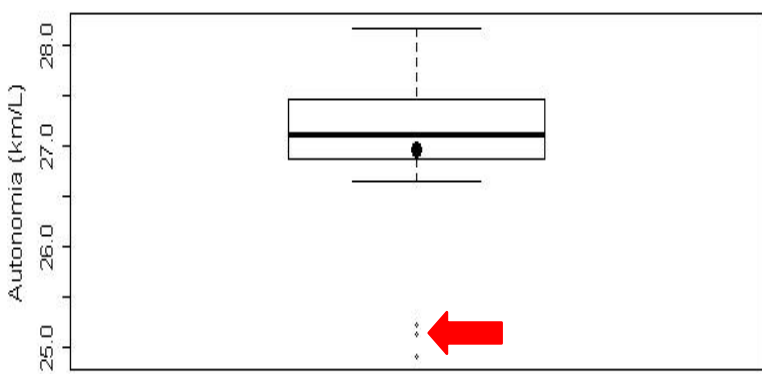

Laboratórios

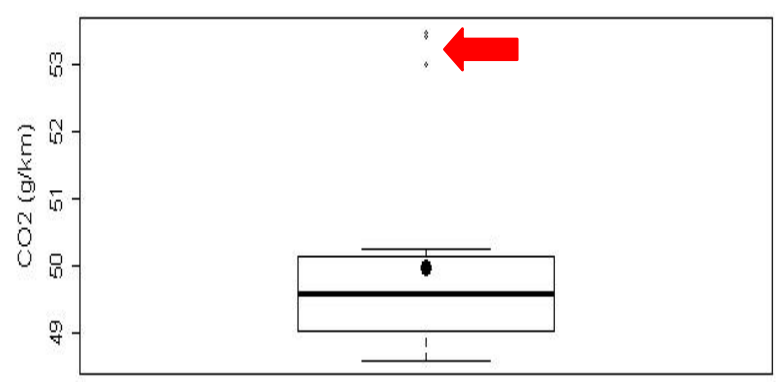

Laboratórios

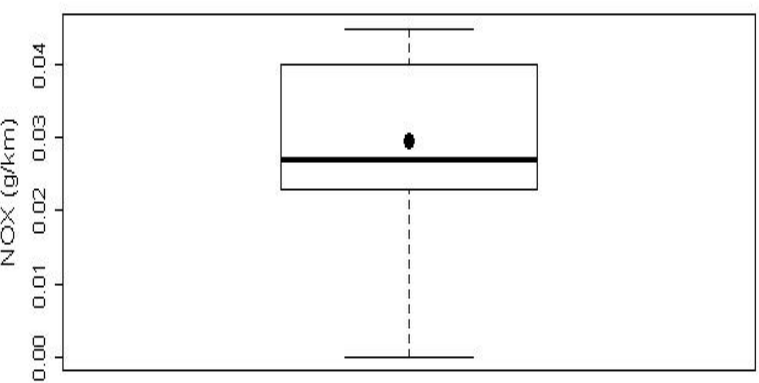

Laboratórios

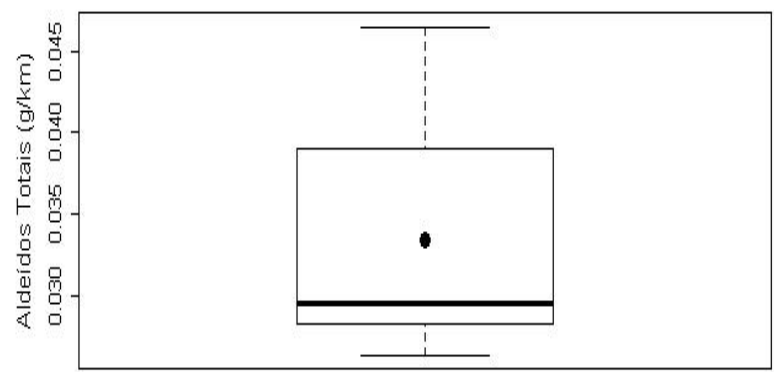

Laboratórios

Figura 4. Box-Plot para o teste de proficiência de emissões em motociclo movido a etanol. (a) $\mathrm{CO}$, (b) $\mathrm{CO}_{2}$, (c) THC, (d) $\mathrm{NO}_{x}$, (e) autonomia e (f) aldeídos totais.

\section{CONCLUSÃO}

Ensaio de proficiência de emissões veiculares é um processo único, conduzido apenas no Brasil. Para esta primeira rodada, os resultados obtidos podem ser considerados extremamente satisfatórios, visto que o EP forneceu informações importantes para que os laboratórios participantes possam analisar criticamente os 
seus processos e fazer as melhorias necessárias. Os laboratórios também poderão dividir as melhores práticas e harmonizar os procedimentos de análise de emissões.

Novas rodadas estão atualmente em andamento, uma delas envolvendo o ciclo PROMOT M4 que estará em vigor a partir de 2014.

\section{AGRADECIMENTOS}

Este trabalho foi desenvolvido junto a Comissão Técnica de Acreditação de Laboratórios de Emissões de Motos da Associação Brasileira de Engenharia Automotiva - AEA.

Agradecemos aos membros da comissão pelas discussões e o total envolvimento na realização dos ensaios de proficiência.

Finalmente agradecemos a Moto Honda da Amazônia e Yamaha Motor da Amazônia pela cessão dos motociclos utilizados na realização deste ensaio de proficiência.

\section{REFERÊNCIAS}

[1] Estatística da frota brasileira. Disponível em: http://www.denatran.gov.br/frota.htm .Acesso em 23/05/2013.

[2] L.F.A. Garcia, S.M. Corrêa, R. Penteado, L.C. Daemme, L. V. Gatti, D. S. Alvim, Measurements of Emissions from Motorcycles and Modeling Its Impact on Air Quality, J. Braz. Chem. Soc., Vol. 24, No. 3, 375-384, 2013.

[3] C.D.R. Souza, S.D. Silva, M. A. V. Silva, M.A. D'agosto, A.P. Barboza, Inventory of conventional air pollutants emissions from road transportation for the state of Rio de Janeiro, Energy Policy, Vol. 53, 125-135, 2013.

[4] INTERNATIONAL ORGANIZATION FOR STANDARDIZATION - ISO/IEC 17043 - conformity assessment - General requirement for proficiency testing. Geneva, 2010.

[5] ISO 5725 (E) - Accuracy (trueness and precision) of measurement methods and results - Part 2: Basic method for the determination of repeatability and reproducibility of a standard measurement method, Geneva,1994.

[6] Relatório Final do Ensaio de Proficiência em Emissões Veiculares - $2^{\underline{a}}$ rodada.

Disponível

http://www.inmetro.gov.br/metcientifica/profiEmiVeicular.asp $\quad$ Acesso em 20/05/2013.

[7] Relatório Final do Ensaio de Proficiência em Emissões Veiculares - 3a rodada.

http://www.inmetro.gov.br/metcientifica/profiEmiVeicular.asp 20/05/2013.

[8] Relatório Final do Ensaio de Proficiência em Emissões Veiculares - $4^{\underline{a}}$ rodada.

Disponível

em http://www.inmetro.gov.br/metcientifica/profiEmiVeicular.asp 20/05/2013.

Acesso em 
[9] Relatório Final do Ensaio de Proficiência em Emissões de motociclos - $1^{\text {a }}$ rodada.

Disponível

em

http://www.inmetro.gov.br/metcientifica/pdf/rf emissoes motociclos 1a rodada.pd f Acesso em 20/05/2013.

[10] NIST/SEMATECH, e-Handbook of Statistical Methods, Disponível em http://www.itl.nist.gov/div898/handbook/, Acesso em 24/05/2013.

[11] Miller, J.C. and Miller, J.N. Statitistics for Analytical Chemistry, $3^{\text {rd }}$ Ed. Ellis Horwood, Chichester, 1993. 